\title{
VALIDACIÓN DE UNA ESCALA DE EMPODERAMIENTO Y AGENCIA PERSONAL EN MUJERES MEXICANAS
}

\author{
Nélida Padilla Gámez y Cinthia Cruz del Castillo \\ FES Iztacala, UNAM; Universidad Iberoamericana \\ México
}

\begin{abstract}
RESUMEN
A lo largo de la historia se observa que las mujeres han vivido y viven en condiciones de mayor desventaja por el solo hecho de haber nacido con este sexo; en roles que son incuestionablemente ejecutados, mismos que obstaculizan una diferenciación o autonomía en su entorno personal y social; pero, ¿existirán algunas situaciones en las cuales las mujeres mexicanas se perciban más autónomas y empoderadas? En tal sentido, el objetivo de esta investigación fue diseñar una escala válida y confiable que contemplara algunas condiciones o situaciones en las que las mujeres se identifiquen con las habilidades de agencia personal para tomar acciones o decisiones tanto en lo personal como en lo social. La escala total mostró niveles adecuados de confiabilidad ( $\alpha=0.92)$ y validez, y el análisis arrojó cuatro factores: la agencia personal, la autoconciencia, el empoderamiento en la salud y el empoderamiento social. Los análisis de diferencias por escolaridad y por edad mostraron que las mujeres jóvenes y con mayor nivel educativo tienen más habilidades de agencia personal y de empoderamiento, y en casi todos los grupos analizados se observó una tendencia a mostrar menos habilidades de empoderamiento en la salud.
\end{abstract}

Palabras Clave:

empoderamiento, mujeres, escala, cultura mexicana.

\section{VALIDATION OF A SCALE OF EMPOWERMENT AND PERSONAL AGENCY IN MEXICAN WOMEN}

\begin{abstract}
Throughout history has been observed that women have lived and live in conditions of greater disadvantage by the mere fact of having been born female; in roles that are unquestionably executed, which impede a differentiation or autonomy in their personal and social environment; but will there be some situations in which mexican women perceive themselves to be more autonomous and empowered? In this sense, the objective of this research was to design a valid and reliable scale that contemplates some conditions or situations in which women identify with the abilities of personal empowerment and agency to take actions or decisions in the personal as in the social. 155 mexican women with 35 years in average answered the overall scale integrated by 50 items with nine Likert options. The final version of the scale with 47 items remaining showed adequate levels of reliability $(\alpha=0.92)$ and validity, the analysis yielded four factors: personal agency, self-awareness, empowerment in health, and social empowerment. Analysis of differences by schooling and age showed that young women with higher levels of education have greater personal agency and empowerment skills, and in almost all groups analyzed, there was a tendency to show less health empowerment skills.
\end{abstract}

\section{Keywords:}

empowerment, women, scale,mexican culture.

Bitácora del Artículo:

| Recibido: 1 de Febrero de 2018 | Aceptado: 1 Abril de 2018 | Publicado en línea: Enero-Junio de 2018 | 


\title{
Autoría y Derechos de Propiedad Intelectual
}

\section{VALIDACIÓN DE UNA ESCALA DE EMPODERAMIENTO Y AGENCIA PERSONAL EN MUJERES MEXICANAS}

\author{
Nélida Padilla Gámez y Cinthia Cruz del Castillo \\ FES Iztacala, UNAM; Universidad Iberoamericana \\ México
}

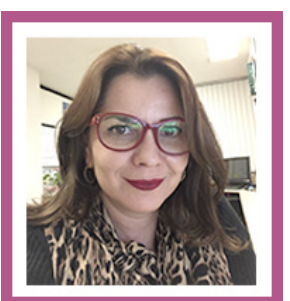

Nélida Padilla Gámez

FES Iztacala, UNAM

Correo: nelidapadillag@gmail.com

Licenciatura y Doctorado en Psicología en la Facultad de Psicología de la UNAM, labora en la FES IZTACALA como profesora asociada "C" T.C. forma parte del grupo de investigación en Psicología y Salud Sexual, docente en la Residencia de Terapia Familiar y en la Licenciatura de Psicología.

Ver más...

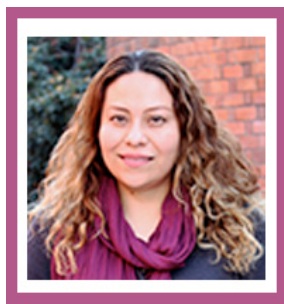

Cinthia Cruz del Castillo Departamento de Psicología, Universidad Iberoamericana.

Correo: cinthia.cruz@ibero.mx

Licenciatura y Doctorado en Psicología por la UNAM. Labora como Profesor e Investigador de tiempo completo y es Coordinadora del Doctorado en Investigación Psicológica en el Departamento de Psicología de la Universidad Iberoamericana, Campus Santa Fe, México.

Ver más...

\section{CONTRIBución DE lOS AutORES}

Las autoras desarrollaron la presente investigación en conjunto, participando en cada fase de la misma.

\section{AGRADECIMIENTOS}

La presente investigación se desarrolló con el apoyo recibido por Estancia de Investigación Posdoctoral del CONACYT.

\section{Datos de FiLIACIÓN DE LAS Autoras}

FES Iztacala, UNAM |Universidad Iberoamericana

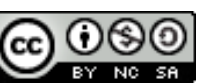

Copyright: (c) 2018 Padilla-Gámez, N. \& Cruz-del Castillo, C.

Este es un artículo de acceso abierto distribuido bajo los términos de la licencia Creative Commons Reconocimiento-NoComercial -Compartirlgual 4.0 Internacional, por lo que su contenido gráfico y escrito se puede compartir, copiar y redistribuir total o parcialmente sin necesidad de permiso expreso de sus autoras con la única condición de que no se puede usar con fines directamente comerciales y los términos legales de cualquier trabajo derivado deben ser los mismos que se expresan en la presente declaración. La única condición es que se cite la fuente con referencia a la Revista Digital Internacional de Psicología y Ciencia Social y a sus autoras. 


\section{TABLA DE CONTENIDO}

INTRODUCCIÓN

MÉTOdo

Participantes, 26

Instrumentos, 26

Procedimiento, 26

Resultados

Discusión

REFERENCIAS 


\section{INTRODUCCIÓN}

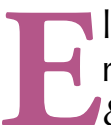
empoderamiento ha sido estudiado sobre todo respecto a las mujeres (Brown, Pecheny, Gattoni \& Tamburrino, 2013; Casique, 2010; Doubova et al., 2013) como consecuencia de que a lo largo de la historia son ellas las que han estado en mayor desventaja y han sido desfavorecidas en todos los contextos, desde lo individual, lo relacional, lo social, lo religioso, lo institucional y lo laboral (Pick, García \& Leenen, 2011; Sayavedra \& Flores, 1997).

El empoderamiento se ha clasificado como intrapersonal e interaccional (Wilke \& Speer, 2011), intrínseco y extrínseco, individual y relacional (Zimmerman, 1995), o como proceso individual y colectivo (Sayavedra \& Flores, 1997). El empoderamiento es un constructo complejo y multifactorial que involucra las capacidades físicas y mentales, la historia personal y las habilidades desarrolladas a lo largo de la vida.

La dimensión individual del empoderamiento hace referencia a la capacidad de tomar decisiones respecto a eventos importantes (Sayavedra \& Flores, 1997), a tener el control de nuestras vidas, a establecer objetivos, a adquirir habilidades, a tener autoconfianza y resolver problemas en lo individual (Varkey, Kureshi \& Lesnick, 2010), teniendo una estrecha relación con el desarrollo humano (Silva \& Loreto, 2004).

Desde lo interaccional, el empoderamiento se ha relacionado con la participación activa dentro de las comunidades (Varkey, Kureshi \& Lesnick, 2010), a tener voz y voto en contextos políticos y sociales (Charmes \& Wieringa, 2003), a la posibilidad de generar recursos materiales (Wilke \& Speer, 2011), de generar estrategias de acción y de comunicación e interrelación con los otros en los procesos colectivos, políticos y sociales (Varkey, Kureshi \& Lesnick, 2010), usando los recursos de su entorno a pesar de la oposición de otras personas o instituciones (Sayavedra \& Flores, 1997). Por esta razón, el término poderío — derivado de empoderamiento, por su traducción literal del inglés empowerment, "empoderamiento" (Sayavedra \& Flores, 1997) - se entiende como un hecho que trasciende al individuo y se plasma en los sujetos y en los espacios sociales.

Existe una diversidad de instrumentos desarrollados para medir el empoderamiento, mismos que se mencionan a continuación; no obstante, ninguna de estas medidas contempla todas las aristas de las que se compone el empoderamiento, y se enfocan a medir — por separado— el empoderamiento individual o comunitario, la inequidad o la salud.

Una de las escalas más utilizadas para medir el empoderamiento es el GEM (Gender Empowerment Measure) (Charmes \& Wierienga, 2003; Varkey, Kureshi \& Lesnick, 2010), que mide tres aspectos: 1) participación económica y toma de decisiones; 2) participación política y toma de decisiones, y 3) poder sobre los recursos económicos. Charmes y Wieringa (2003) identifican dos dimensiones respecto al empoderamiento. La primera lo describe como un campo de operación, sus dimensiones, sus interrelaciones, y sus interseccionalidades con otros campos, así como esos de raza/etnia y clase. La segunda dimensión plantea que el empoderamiento en las mujeres es un proceso que considera elementos como la conciencia, la elección/alternativas, los recursos, la voz, la agencia y la participación. Esta dimensión del empoderamiento femenino está relacionada con la mejora en habilidades de toma de decisiones en las áreas fundamentales de sus vidas. También desarrollaron una medida (matriz) de empoderamiento con perspectiva de género que denominaron WEM (Women's Empowerment Matrix). Fue diseñada para revisar un mapa general del género, sus aplicaciones y sus interrelaciones en otros temas como la educación, el trabajo de las mujeres y VIH/SIDA. Esta medida invita a visualizar un proyecto o programa, y a su vez contemplar varios ámbitos de interconexión entre varias esferas o temas que se relacionan con las acciones de empoderamiento. Las esferas que distingue son la física, sociocultural, religiosa, política, económica y legal, que van de lo individual a lo general. Es útil para las personas que desean investigar un área en particular y encontrar interrelaciones con otras áreas; la fortaleza de esta propuesta radica en la posibilidad de generar teorías y proyectos basados en la identificación de áreas de oportunidad y desarrollar programas de trabajo con ellas. Sin embargo, es más una propuesta exploratoria que de medición. Ya Wilke y Speer (2011) se planteaban la necesidad de identificar los procesos intrapersonales (emocionales) del empoderamiento porque la mayoría de las investigaciones desarrolladas han analizado el constructo del empoderamiento psicológico, dejando a un lado los componentes intrapersonales e interaccionales del empoderamiento. El componente intrapersonal ha sido descrito como la manera en la cual los individuos piensan respecto a sí mismos y sus capacidades de tener éxito en el mundo, incluida la noción de tener control, autoeficacia y percibirse competente.

Por otra parte, el empoderamiento visto desde una perspectiva psicopolítica tiene significados más profundos, pues se asume que — frente a la creencia generali- 
zada de que es algo lejano que detentan los otros, los poderosos- el poder, como potencial a realizar en el ser personal y comunitario, reside en el individuo mismo, en la gente común, y se puede hacer efectivo si se tiene conciencia de ese potencial genérico, la gente o la comunidad actúan eficazmente para hacerlo realidad. Esa — según Sánchez (2013) — es la clave, y es posible resumirla afirmando que el poder es, cuando menos, un medio esencial para el desarrollo humano.

El empoderamiento está vinculado con la autonomía — también conocida como autoeficacia o agencia personal (Sánchez Vidal, 2013; Wilke \& Speer, 2011; Varkey, Kureshi \& Lesnick, 2010; Pick, Sirkin, Ortega, Osorio, Martínez, Xocolotzin \& Guivaudan, 2007; Charmes \& Wieringa, 2003; Sayavedra \& Flores, 1997)—. La agencia personal se ha definido en psicología, filosofía y en el ámbito del desarrollo socioeconómico como una manera de explicar aspectos del funcionamiento humano autónomo (Bandura, 1996, citado en Pick, Sirkin, Ortega, Osorio, Martínez, Xocolotzin \& Guivaudan, 2007; Bandura, 2005). Este concepto hace referencia a la competencia personal, enfocándose sobre todo en el individuo como un actor que se desarrolla en su contexto social. Asimismo, la agencia personal es la habilidad de definir los objetivos propios de manera autónoma y de actuar a partir de los mismos: "lo que una persona tiene libertad de hacer y lograr en búsqueda de los objetivos o valores que el individuo considere importantes" (Sen, 1985: 203). Por tanto, este concepto incluye, además de la acción, la intención, el significado, la motivación, así como el propósito que los individuos imprimen a sus actividades. La expresión de la agencia es en última instancia más que una conducta o decisión, ya que incorpora metas, objetivos, acuerdos, obligaciones (Sen, 1985). Implica la capacidad de predicción y control que la persona tiene sobre las elecciones, decisiones y acciones de las cuales es responsable.

La agencia se construye con los recursos que los individuos o los grupos poseen. La gente puede tener recursos materiales, como el capital financiero y productivo, y recursos no materiales, como las habilidades, el conocimiento, las redes sociales y la capacidad psicológica para aspirar e imaginar el cambio (Alsop \& Heinsohn, 2005, citado en Pick et al., 2007). La agencia como la capacidad de autogobierno, un componente de la autonomía, y para fines de medición, la han dividido en "autonomía de actitudes", "autonomía emocional" y "autonomía funcional" (Beyers, Groossens, Vansant \& Moors, 2003).

Bandura (2000) define agencia en tres partes: "agencia personal, agencia por delegación, en la que se depende de otros para actuar a instancias propias con la finalidad de ob- tener los resultados deseados, y agencia colectiva, la cual se ejerce a través de un esfuerzo socialmente coordinador e interdependiente", y concibe la agencia como algo que ocurre en un contexto donde "las personas son productoras, además de los productos, de sistemas sociales".

La agencia es difícil de medir porque a menudo se comporta de diversas formas; puede observarse en la toma de decisiones, o en la negociación, asertividad o en otros procesos de reflexión y análisis (Pick et al., 2007; Casique, 2010).

Uno de los pasos más importantes del empoderamiento en las mujeres es cuando adquieren agencia personal, es decir, cuando actúan a su favor. Esto implica una intervención significativa y útil en la construcción de algo nuevo. La agencia se da a un nivel personal en las mujeres, cuando luchan por conseguir educación, o comienzan una empresa o proyecto, o cuando se resisten a casarse con quien no quieren (Charmes \& Wieringa, 2003).

De tal forma, el empoderamiento y la agencia personal no son sólo conceptos operativos, sino que también poseen un valor básico en el campo comunitario, y en consecuencia la base de un cambio cultural potencialmente revolucionario al alcance de la psicología. En este tenor, es por medio de la psicología que surge la posibilidad de cambiar la concepción que los humanos tenemos de nosotros mismos (generando nuevos modos de actuar, pensar, sentir), elevándonos a la calidad de sujetos agentes de nuestros propios objetivos y proyectos humanos, en lugar de ser servidores, agentes de otros.

Lo anterior se muestra como el significado ideológico del empoderamiento que, como se ha indicado, fundamenta en psicología la idea y práctica del desarrollo humano, y en el campo social la acción social y política desde abajo, como complemento de la acción formal, desde arriba (Fonseca \& Quintero, 2008).

De nada sirve promover la intuición de que la gente tiene un potencial "endógeno" de poder para desarrollarse personalmente y alcanzar la igualdad social, si no se pueden promover procedimientos y estrategias eficaces para hacer realidad ese potencial. Este es precisamente el reto y el riesgo de la psicología, en particular de la comunitaria (Sánchez, 2013).

Con base en lo anterior, y en un intento de plantear un continuo entre la vulnerabilidad y el empoderamiento en las mujeres, se desarrolló un inventario que contempla los factores más importantes asociados con el empoderamiento, como salud, familia, pareja, hijos, enfermedades, toma de decisiones, comunidad, etcétera. Por tanto, el objetivo de la presente investigación es el diseño de un inventario de empoderamiento y agencia personal, misma que se describe a continuación, e identificar cómo el constructo puede variar por la edad, el número de hijos, tener o no tener pareja, y la escolaridad, porque se ha 
reconocido que estas variables podrían hacer diferencias en los niveles de empoderamiento.

Para fines de esta investigación, se concibe al empoderamiento como una herramienta de desarrollo humano que contempla entre sus componentes el que los individuos sean autónomos, adquieran agencia personal para poderla reflejar en autoeficacia de poder lograr los objetivos planteados.

\section{MÉtodo}

\section{Participantes}

La presente investigación se realizó con una muestra no probabilística intencional de 155 mujeres con edades entre los 18 y 69 años; la media de edad fue de 35 años. La escolaridad promedio fue de licenciatura con $49 \%$. Respecto al estado civil las mujeres reportaron ser solteras en su mayoría. En cuanto a la religión, 75\% refiere ser católica. De las que reportaron tener hijos, 20\% refirió tener dos hijos, mientras que más de la mitad de la muestra dijo no tenerlos. De las ocupaciones más referidas se encontró que $29 \%$ eran estudiantes, $21 \%$ profesionistas y otro $21 \%$ reportó tener un trabajo asalariado; $18 \%$ indicó que se dedicaban al hogar. Por último, en cuanto a la orientación sexual predominó la heterosexualidad (94\%). El instrumento se aplicó a todas las mujeres dispuestas a colaborar en la investigación, en sus centros de estudio, trabajo, hogar y espacios públicos.

\section{Instrumentos}

Este instrumento se deriva de un estudio previo acerca de los aspectos y condiciones que sitúan a las mujeres en condiciones de vulnerabilidad (Padilla Gámez \& Cruz del Castillo, 2014). En este sentido se propone encontrar un eje continuo donde las mujeres puedan identificar en una escala Likert de 9 puntos que mide, en un continuo de 0 a 9 , situaciones en que las mujeres perciben mayor o menor empoderamiento, es decir, mayor agencia personal en la toma de decisiones ante situaciones o condiciones de vulnerabilidad. El formato de respuesta se retoma de la escala de autoeficacia en el climaterio (Doubova, Espinosa, Infante, Aguirre, Rodríguez, Olivares \& Pérez, 2013), misma que se describe a continuación: 0 = no aplica para mí; 1 a 3 = estoy absolutamente segura que no puedo; 4 a $6=$ a veces no podría y a veces sí podría, y 7 a 9 = estoy absolutamente segura que sí puedo hacer algo en diferentes situaciones.

La escala consta de 50 reactivos que abordan situaciones o condiciones de vulnerabilidad o empoderamiento, considerando que 0 refiere que dicha condición no aplica para la persona; de 1 a 3 se percibe poco em- poderamiento para hacer frente a dicha situación y de 4 a 9 se percibe más posibilidades de hacer frente ante la situación. Algunos ejemplos de reactivos son "Exijo mis derechos aunque otros no estén de acuerdo", "Puedo hacer algo ante la situación de tener un familiar enfermo", "Puedo conseguir un trabajo remunerado", "Es mejor tomar decisiones que esperar a ver lo que pasa", "Puedo hacer algo para evitar el abuso físico", etcétera.

\section{Procedimiento}

Para efectuar la presente investigación se aplicó dicha escala a mujeres mayores de edad, que aceptaron participar en la investigación; se contemplaron a todas las participantes sin importar raza, etnia, situación laboral o escolar, condición socioeconómica, etcétera. La aplicación se hizo en lugares públicos, espacios de trabajo, escuelas, y con la técnica bola de nieve.

Posterior a la aplicación y captura de los instrumentos se hicieron los análisis estadísticos para la obtención de la confiabilidad y validez de la escala; los resultados se describen a continuación.

\section{Resultados}

Para obtener la validez de constructo del instrumento se hizo un análisis factorial exploratorio de componentes principales con rotación ortogonal de los 50 ítems. El análisis factorial arrojó cuatro factores, con valores propios iguales a 1, y 47 reactivos con cargas superiores a 0.30; en conjunto los cuatro factores explican $43.36 \%$ de la varianza total.

Posterior al análisis factorial se logró la confiabilidad de los 47 reactivos obteniendo un alpha de cronbach de $\alpha=0.92$. Los índices de consistencia interna variaron entre 0.77 (empoderamiento social ) y 0.88 (agencia personal). La media más baja fue en el factor de empoderamiento en la salud $(M=5.2)$ y la más alta en el factor de autoconciencia $(M=7.5)$ (tabla 1).

Tabla 1.

Número de reactivos, coeficientes alpha de cronbach, medias y desviaciones para cada factor.

\begin{tabular}{|c|c|c|c|c|}
\hline FACTOR & $\begin{array}{l}\text { Número } \\
\text { DE } \\
\text { REACTIVOS }\end{array}$ & $\alpha$ & M & SD \\
\hline 1. Agencia personal & 16 & 0.88 & 7.3 & 1.40 \\
\hline 2. Autoconciencia & 11 & 0.84 & 7.5 & 1.07 \\
\hline $\begin{array}{l}\text { 3. Empoderamiento en la } \\
\text { salud }\end{array}$ & 12 & 0.86 & 5.2 & 2.20 \\
\hline 4. Empoderamiento social & 8 & 0.77 & 7.1 & 1.30 \\
\hline
\end{tabular}


El factor 1 (agencia personal) se refiere a la capacidad del individuo — en este caso de las mujeres - de tomar sus propias decisiones en torno a situaciones de la vida. A su vez, se considera como la libertad de actuar en función de lo que se necesita y se desea en diversos contextos, ya sea en lo individual (metas personales, salud, sexualidad, etcétera), en lo interpersonal (pareja, familia, hijos, amistades, etcétera), así como en lo social (trabajo, sociedad, etcétera). El factor 2 (autoconciencia) contiene reactivos que se refieren a la capacidad de los individuos de actuar en situaciones de incomodidad o inestabilidad consigo mismo o con el contexto; no obstante, para este factor es indispensable el autoconocimiento para identificar las necesidades, deseos, metas, etcétera, que se pretenden lograr en pro del desarrollo de la agencia personal. El factor 3 (empoderamiento en la salud) agrupa las situaciones y condiciones en que las mujeres pueden o no hacer algo respecto a la salud propia o de su contexto interpersonal y social. Son situaciones que rebasan y pueden poner a las mujeres en condiciones de vulnerabilidad, de no tomar las acciones necesarias. En el factor 4 (empoderamiento social) se observa una tendencia a tomar decisiones en función de su entorno personal e interaccional, para lo cual es importante contar con redes de apoyo, tener claridad en la toma de decisiones en sus relaciones interpersonales para poder expresar las necesidades o desacuerdos a quien sea (tabla 2).

Tabla 2.

Matriz de factores extraidos por rotación varimax y cargas factoriales de los ítems.

\section{Reactivos}

CARGA

FACTORIAL

FACTOR 1. AGENCIA PERSONAL

\begin{tabular}{|l|l|}
\hline $\begin{array}{l}\text { Puedo tomar decisiones sobre lo que me gusta } \\
\text { y no en la sexualidad. }\end{array}$ & 0.77 \\
\hline $\begin{array}{l}\text { Puedo hacer algo para satisfacer mis } \\
\text { necesidades y deseos sexuales. }\end{array}$ & 0.69 \\
\hline $\begin{array}{l}\text { Puedo hacer algo ante un rompimiento } \\
\text { amoroso. }\end{array}$ & 0.65 \\
\hline Puedo hacer algo para evitar el acoso laboral. & 0.64 \\
\hline $\begin{array}{l}\text { Puedo hacer algo cuando me siento poco } \\
\text { aceptada. }\end{array}$ & 0.64 \\
\hline $\begin{array}{l}\text { Puedo hacer algo para cambiar mi condición } \\
\text { laboral. }\end{array}$ & 0.63 \\
\hline Puedo hacer algo para evitar el abuso físico. & 0.62 \\
\hline $\begin{array}{l}\text { Puedo hacer algo para evitar vivir violencia de } \\
\text { pareja. }\end{array}$ & 0.54 \\
\hline
\end{tabular}

Reactivos

Puedo hacer algo para evitar el abuso emocional.

0.52

Yo puedo conseguir un trabajo remunerado económicamente.

0.52

Puedo hacer algo cuando me siento incómoda con mi sexualidad.

0.50

Puedo hacer algo cuando me siento incómoda con mi cuerpo.

0.49

Puedo hacer algo si detecto que tengo una infección de transmisión sexual (ITS).

0.45

Exijo mis derechos aunque otros no estén de acuerdo.

Puedo hacer algo para mantener una relación de pareja.

Puedo decidir si quiero tener hijos.

Puedo decidir si quiero tener hijos.

FACTOR 2. AUTOCONCIENCIA

Puedo hacer algo cuando me siento insegura.

Puedo hacer algo cuando me siento mal conmigo misma.

Puedo hacer algo cuando me siento estresada.

Si tengo un problema puedo solucionarlo.

Puedo hacer algo cuando percibo que tengo más responsabilidades de las que considero me corresponden.

Puedo hacer algo con mis alteraciones en el peso (obesidad, sobrepeso, bajo peso, etcétera).

Puedo hacer algo para cambiar mi condición económica.

Puedo hacer algo cuando me veo fea o poco agraciada.

0.54

Busco la solución a un problema, aunque otros me digan que no hay.

Puedo hacer algo para evitar vivir en condiciones de adversidad y/o pobreza.

Hago lo que creo que es mejor para mí sin importar lo que otros crean.

0.63

0.62

\begin{tabular}{|l|c|}
\hline \multicolumn{2}{|c|}{ FACTOR 3. EMPODERAMIENTO EN LA SALUD } \\
\hline $\begin{array}{l}\text { Puedo hacer algo con mis problemas de } \\
\text { Consumo de drogas. }\end{array}$ & 0.74 \\
\hline $\begin{array}{l}\text { Puedo hacer algo con mis problemas de } \\
\text { alcoholismo. }\end{array}$ & 0.74 \\
\hline $\begin{array}{l}\text { Puedo hacer algo si vivo con alguna } \\
\text { discapacidad física. }\end{array}$ & 0.73 \\
\hline Puedo hacer algo si vivo con diabetes. & 0.70 \\
\hline
\end{tabular}




\begin{tabular}{|c|c|}
\hline Reactivos & $\begin{array}{l}\text { CARGA } \\
\text { FACTORIAL }\end{array}$ \\
\hline Puedo hacer algo con mi depresión. & 0.58 \\
\hline Puedo decidir trabajar en el servicio doméstico. & 0.54 \\
\hline $\begin{array}{l}\text { Puedo hacer algo ante la situación de no poder } \\
\text { tener hijos. }\end{array}$ & 0.51 \\
\hline Puedo decidir trabajar como sexoservidora. & 0.48 \\
\hline $\begin{array}{l}\text { Puedo hacer algo al respecto de mi enfermedad } \\
\text { (cualquiera que sea). }\end{array}$ & 0.46 \\
\hline $\begin{array}{l}\text { Puedo hacer algo ante la situación de tener un } \\
\text { familiar con problemas de drogadicción }\end{array}$ & 0.46 \\
\hline $\begin{array}{l}\text { Puedo hacer algo ante un embarazo no } \\
\text { deseado. }\end{array}$ & 0.45 \\
\hline $\begin{array}{l}\text { Puedo hacer algo ante la situación de tener un } \\
\text { familiar con problemas de alcoholismo. }\end{array}$ & 0.39 \\
\hline \multicolumn{2}{|l|}{ FACTOR 4. EMPODERAMIENTO SOCIAL } \\
\hline $\begin{array}{l}\text { Puedo hacer algo para contar con redes de } \\
\text { apoyo cuando lo necesito. }\end{array}$ & 0.70 \\
\hline $\begin{array}{l}\text { Puedo expresar mis necesidades abiertamente } \\
\text { a quien sea. }\end{array}$ & 0.63 \\
\hline $\begin{array}{l}\text { Es mejor tomar decisiones que esperar a ver lo } \\
\text { que pasa. }\end{array}$ & 0.60 \\
\hline $\begin{array}{l}\text { Me quejo con las autoridades cuando hay un } \\
\text { abuso. }\end{array}$ & 0.58 \\
\hline $\begin{array}{l}\text { Puedo expresar abiertamente mis desacuerdos } \\
\text { a quien sea. }\end{array}$ & 0.48 \\
\hline $\begin{array}{l}\text { Puedo hacer algo si detecto que tengo Virus del } \\
\text { Papiloma Humano (VPH) }\end{array}$ & 0.46 \\
\hline $\begin{array}{l}\text { Puedo hacer algo para evitar violencia } \\
\text { intrafamiliar. }\end{array}$ & 0.37 \\
\hline $\begin{array}{l}\text { Puedo tomar decisiones sobre mi orientación } \\
\text { sexual. }\end{array}$ & 0.36 \\
\hline
\end{tabular}

Con la finalidad de observar si existen diferencias en los cuatro factores de la escala y la edad de las mujeres, y dado que la edad varía de 26 a 69 años, se dividió la muestra en tres grupos de edad: grupo 1, mujeres de 18 a 25 años, grupo 2, mujeres de 26 a 42 años, y grupo 3, mujeres de 43 a 69 años; se realizó un análisis de varianza simple (tabla 3 ).

En términos generales las mujeres del grupo 2 (2642 años) tienen la media más alta en el factor de agencia personal, autoconciencia y empoderamiento social. En cuanto al factor de empoderamiento en salud fue el grupo 1 (18-25 años) el que presentó la media más alta, en contraste con las mujeres del grupo 3 (43-69 años), que en temas de salud muestran menos habilidades de agen- cia personal y empoderamiento para resolver o enfrentar condiciones adversas que las rebasan y vulneran.

Tabla 3.

Análisis de varianza simple entre los cuatro factores de la escala de empoderamiento y los tres grupos de edad.

\begin{tabular}{|c|c|c|c|c|c|c|c|c|}
\hline \multirow[t]{2}{*}{ FACTOR } & \multicolumn{2}{|c|}{$\begin{array}{c}\text { GRUPO } 1 \\
18-25 \\
\text { AÑos }\end{array}$} & \multicolumn{2}{|c|}{$\begin{array}{c}\text { GRUPO } 2 \\
26-42 \\
\text { AÑos }\end{array}$} & \multicolumn{2}{|c|}{$\begin{array}{c}\text { GRUPO } 3 \\
43-69 \\
\text { AÑOS }\end{array}$} & \multirow[t]{2}{*}{ F } & \multirow[t]{2}{*}{$P$} \\
\hline & M & SD & M & SD & M & SD & & \\
\hline Agencia personal & 7.5 & 1.10 & 7.8 & 0.99 & 6.6 & 1.70 & 10.80 & 0.00 \\
\hline Autoconciencia & 7.4 & 1.20 & 7.8 & 0.82 & 7.4 & 0.98 & 2.43 & 0.09 \\
\hline $\begin{array}{l}\text { Empoderamiento } \\
\text { en la salud }\end{array}$ & 6.1 & 2.01 & 5.3 & 2.20 & 4.3 & 2.08 & 9.02 & 0.00 \\
\hline $\begin{array}{l}\text { Empoderamiento } \\
\text { social }\end{array}$ & 7.3 & 1.20 & 7.5 & 0.95 & 6.5 & 1.50 & 8.65 & 0.00 \\
\hline
\end{tabular}

En términos generales se observó que los grupos 1 y 2 muestran tener más herramientas de agencia personal y de empoderamiento que las mujeres del grupo 3, tal vez debido a que los dos primeros grupos se encuentran en la etapa de mayor productividad, además de que pertenecen a otra generación más reciente en donde se ha promovido que las mujeres tomen decisiones y sean autónomas e independientes, que con las mujeres del tercer grupo que vivieron en la transición de este cambio social derivado de los movimientos feministas.

Las que toman más responsabilidades de las cuestiones asociadas con su propia salud, así como con las condiciones de salud de su contexto inmediato, llámese pareja o familia.

Por otra parte, también se realizó una prueba t de Student para identificar las diferencias entre las medias de los cuatro factores y los grupos de mujeres que reportaron tener hijos y las que no (tabla 4).

\section{Tabla 4.}

Prueba t de Student para muestras independientes entre los cuatro factores de la escala de empoderamiento por el grupo de mujeres que tienen hijos y el grupo de mujeres que no los tienen.

\begin{tabular}{|c|c|c|c|c|c|c|}
\hline \multirow[t]{2}{*}{ FACTOR } & \multicolumn{2}{|c|}{$\begin{array}{l}\text { No tIENE } \\
\text { HIJOS }\end{array}$} & \multicolumn{2}{|c|}{ Tiene hiJOS } & \multirow[t]{2}{*}{$T$} & \multirow[t]{2}{*}{$P$} \\
\hline & $M$ & SD & $M$ & SD & & \\
\hline Agencia personal & 7.7 & 1.06 & 6.9 & 1.6 & 3.39 & 0.001 \\
\hline Autoconciencia & 7.5 & 1.10 & 7.5 & 1.0 & 0.14 & 0.88 \\
\hline $\begin{array}{l}\text { Empoderamiento en } \\
\text { la salud }\end{array}$ & 5.8 & 2.10 & 4.5 & 2.0 & 3.76 & 0.00 \\
\hline $\begin{array}{l}\text { Empoderamiento } \\
\text { social }\end{array}$ & 7.4 & 1.10 & 6.9 & 1.5 & 2.29 & 0.024 \\
\hline
\end{tabular}


En este sentido, se encontró que las mujeres que no tienen hijos tienen medias más altas en los factores de agencia personal, de empoderamiento en la salud y de empoderamiento social, lo que puede indicar que tener hijos requiere y demanda tiempo, esfuerzo y recursos, que de no tener hijos dichos recursos se tienen sólo para sí mismas. En el factor de empoderamiento en la salud, es importante destacar que es de los factores con medias más bajas en comparación con los demás factores.

Para conocer si las diferencias en las medias por emparejamiento en los cuatro factores de las escalas fueron significativas, se corrió una prueba t de Student para muestras independientes (tabla 5).

\section{Tabla 5.}

Prueba $t$ de Student para muestras independientes entre los cuatro factores de la escala de empoderamiento y los grupos de mujeres que no tienen y que tienen pareja.

\begin{tabular}{|c|c|c|c|c|c|c|}
\hline \multirow[t]{2}{*}{ FACTOR } & \multicolumn{2}{|c|}{$\begin{array}{l}\text { No tiene } \\
\text { PAREJA }\end{array}$} & \multicolumn{2}{|c|}{ TIENE PAREJA } & \multirow[t]{2}{*}{$\mathrm{T}$} & \multirow[t]{2}{*}{$P$} \\
\hline & M & SD & $M$ & SD & & \\
\hline Agencia personal & 7.6 & 1.30 & 6.9 & 1.50 & 2.62 & 0.010 \\
\hline Autoconciencia & 7.6 & 1.09 & 7.4 & 1.08 & 0.90 & 0.350 \\
\hline $\begin{array}{l}\text { Empoderamiento en } \\
\text { la salud }\end{array}$ & 5.6 & 2.10 & 4.7 & 2.10 & 2.47 & 0.015 \\
\hline $\begin{array}{l}\text { Empoderamiento } \\
\text { social }\end{array}$ & 7.4 & 1.20 & 6.8 & 1.30 & 2.79 & 0.006 \\
\hline
\end{tabular}

Se encontraron diferencias significativas en los factores de agencia personal, empoderamiento en la salud y en empoderamiento social, mostrando que las mujeres que no tienen pareja tienen medias más altas respecto a las mujeres que sí tienen, quizá debido a que las mujeres que sí tienen pareja requieren de negociar, aceptar y sobrellevar situaciones que las mujeres que viven sin pareja. De igual modo se observó que en el factor de empoderamiento en la salud se tienen diferencias significativas entre los dos grupos con medias bajas en comparación con los demás factores, aunado a que las mujeres que sí tienen pareja muestran menos toma de decisiones y empoderamiento en este rubro.

Respecto a la escolaridad, se hizo un análisis de varianza simple entre los factores de escala de empoderamiento y los grupos de educación básica y educación media superior con el grupo de educación superior y posgrado (tabla 6), mostrando las dos últimas medias más altas en los factores de empoderamiento en comparación con el grupo de educación básica. De igual manera, cabe destacar que en el rubro de empoderamiento en la salud las medias fueron bajas y las diferencias encontradas se comportaron igual que con los demás factores.

Tabla 6.

Análisis de varianza simple entre los cuatro factores de la escala de empoderamiento y los grupos de escolaridad.

\begin{tabular}{|c|c|c|c|c|c|c|}
\hline \multirow{2}{*}{ FACTOR } & \multicolumn{2}{|c|}{$\begin{array}{l}\text { EduCACIÓN } \\
\text { BÁSICA }\end{array}$} & \multicolumn{2}{|c|}{$\begin{array}{c}\text { EDUCACIÓN } \\
\text { MEDIA SUPERIOR }\end{array}$} & \multirow[t]{2}{*}{$F$} & \multirow[t]{2}{*}{$P$} \\
\hline & M & SD & $\mathbf{M}$ & SD & & \\
\hline Agencia personal & 6.1 & 1.90 & 6.8 & 1.50 & 9.234 & 0.050 \\
\hline Autoconciencia & 7.1 & 1.04 & 7.3 & 1.10 & 2.099 & 0.103 \\
\hline $\begin{array}{l}\text { Empoderamiento } \\
\text { en la salud }\end{array}$ & 4.2 & 1.70 & 4.2 & 2.03 & 4.932 & 0.050 \\
\hline $\begin{array}{l}\text { Empoderamiento } \\
\text { social }\end{array}$ & 6.3 & 1.50 & 6.4 & 1.60 & 8.392 & 0.050 \\
\hline
\end{tabular}

\begin{tabular}{|c|c|c|c|c|c|c|}
\hline \multirow[t]{2}{*}{ FACTOR } & \multicolumn{2}{|c|}{$\begin{array}{l}\text { EDUCACIÓN } \\
\text { SUPERIOR }\end{array}$} & \multicolumn{2}{|c|}{ Posgrado } & \multirow[t]{2}{*}{$F$} & \multirow[t]{2}{*}{$P$} \\
\hline & M & SD & M & SD & & \\
\hline Agencia personal & 7.6 & 1.10 & 7.9 & 0.69 & 9.234 & 0.050 \\
\hline Autoconciencia & 7.6 & 1.10 & 7.8 & 0.86 & 2.099 & 0.103 \\
\hline $\begin{array}{l}\text { Empoderamiento } \\
\text { en la salud }\end{array}$ & 5.6 & 2.20 & 5.7 & 2.1 & 4.932 & 0.050 \\
\hline $\begin{array}{l}\text { Empoderamiento } \\
\text { social }\end{array}$ & 7.4 & 1.09 & 7.7 & 1.06 & 8.392 & 0.050 \\
\hline
\end{tabular}

Así, las diferencias encontradas en el factor agencia personal versaron entre los grupos de educación básica, educación superior y posgrado; el promedio mayor lo obtuvo el grupo de posgrado (7.9) seguido del grupo de educación superior (7.6), y donde la educación básica muestra el promedio más bajo en cuanto agencia personal (6.1). Por su parte, en el factor empoderamiento en la salud mostró diferencias significativas entre los grupos de educación media superior y superior, en donde el grupo de educación superior tiene un promedio más alto (5.6) en comparación con el grupo de educación media superior (4.2). En el factor empoderamiento social los grupos de educación superior (7.4) y posgrado (7.7) mostraron promedios más altos en relación con el grupo de educación media superior (6.4). Lo anterior muestra lo referido en la literatura acerca de la importancia de la educación en los procesos de empoderamiento. Con una escolaridad más baja, se tiene menos acceso a la adquisición de recursos tanto personales como sociales. Aquí radica la tan importante tarea de promover habilidades de empoderamiento en las mujeres en todos los ámbitos académicos y de todas las edades, pero en especial en las que se encuentran en condiciones de mayor vulnerabilidad. 
Finalmente se efectuó una serie de correlaciones producto momento de Pearson intrafactor con la presencia de hijos y la edad. Los resultados obtenidos muestran una fuerte y significativa relación entre tener hijos y la edad $(r=0.700, p<0.01)$ para el empoderamiento.

Por otra parte, para los factores de agencia personal $(r=-0.399, p<0.01)$ y empoderamiento en la salud $(r=$ -0.345, $p<0.01)$ se observaron relaciones negativas con tener hijos $(r=-0.268, p<0.01)$, lo cual indica que la mujeres que tienen hijos refieren tener menos estrategias y habilidades de agencia personal, así como menos empoderamiento en la salud, esto debido a que las tareas de crianza y trabajo en el hogar - tal vez doble jornada - suscita que las mujeres no atiendan su salud ni puedan tomar decisiones que mejoren sus condiciones, lo cual se refleja en las pocas oportunidades de verse, cuidarse o trabajar en su crecimiento personal. Asimismo se encontraron correlaciones negativas con la edad $(r=-0.272, p<0.01)$, los hijos $(r=-0.194, p<0.05)$ y el empoderamiento social.

En este sentido se observa que la edad se relaciona de modo negativo con la posibilidad de participar en la comunidad y en la toma de decisiones propias y con el entorno, lo cual se confirma con las diferencias encontradas que a mayor edad menos habilidades de agencia personal y empoderamiento, en este caso, con el contexto. Por su parte, tener hijos también se relaciona negativamente con el empoderamiento social, quizá debido a la tarea tan absorbente del maternaje, que impide atender otras actividades en su beneficio, así como en la comunidad.

Por otra parte, se encontró una relación significativa entre los factores de autoconciencia $(r=0.572, p<$ $0.01)$, empoderamiento en la salud $(r=0.522, p<0.01)$ $y$ el empoderamiento social $(r=0.576, p<0.01)$. Esto refleja y coincide con lo referido en la literatura, donde la agencia personal tiene componentes de autoconocimiento, cuidado de la salud, de sí misma, claridad en las metas y proyectos de vida, toma de decisiones activa y consiente tanto a nivel individual como social.

Un resultado interesante fue encontrar que el empoderamiento en la salud mostró una correlación significativa, pero baja en comparación con los demás factores $(r=0.359, p<0.01)$, y algo de llamar la atención fue la ausencia de correlación entre autoconciencia y el empoderamiento en la salud. De modo que para poder tomar decisiones activas respecto al cuidado de la salud es necesario el autoconocimiento, y el atender las necesidades propias antes que las de los demás. En este sentido, se repite la tendencia de muchas mujeres en donde la salud tiene el último lugar en las prioridades de su vida. Se esperaría que a mayor agencia personal se tuvieran más habilidades de empoderamiento en la salud; sin embargo esto no sucedió al menos en este estudio; en este sentido, como lo refiere la literatura, el cuerpo de las mujeres es y vive en función de los otros más que para sí misma (Cobo, 2011).

\section{DisCusión}

Con base en los resultados obtenidos, se observa que la escala de empoderamiento y agencia personal para mujeres tiene adecuados niveles de validez y confiabilidad. Cumple con los objetivos de la presente investigación, que fue el diseño de un instrumento que contemplara multifactorialmente los aspectos involucrados en la agencia personal y el empoderamiento, del cual se desprenden cuatro factores: agencia personal, autoconciencia, empoderamiento en la salud y empoderamiento social, que pueden coadyuvar a identificar, entre estas áreas, las de mayor vulnerabilidad.

De los resultados obtenidos por grupos de edad, escolaridad, emparejamiento, y la presencia o ausencia de hijos, lo que más destaca es la tendencia a encontrar medias bajas, así como correlaciones negativas en el factor de empoderamiento en la salud, con el aumento de edad, presencia de hijos y mujeres que tienen pareja. Las mujeres más jóvenes y de edad adulta mostraron tener más habilidades de empoderamiento; no obstante, en la salud se mantuvo la constante ya referida.

Cabe mencionar que una de las áreas donde se observan mayores índices de vulnerabilidad es en el área de la salud y lo que conlleva, es decir, malestares permanentes, enfermedades y padecimientos evitables, mala atención, sumándose el desgaste físico y subjetivo debido a la sobrecarga de actividades, al descuido de su cuerpo, malos tratos y violencia de todo tipo (Sayavedra \& Flores, 1997).

En México las mujeres en etapa de climaterio tienden a adoptar un estilo de vida poco saludable, caracterizado por el sedentarismo, insuficiente ingesta de frutas, verduras y alimentos ricos en calcio, y baja utilización de programas de detección oportuna de enfermedades crónicas, circunstancias que las hacen más propensas a desarrollar enfermedades (Doubova, Espinosa, Infante, Aguirre, Rodríguez, Olivares \& Pérez, 2013).

Este panorama no es específico de las pobres, explotadas y marginadas, aunque en ellas hace estragos la doble opresión, y en el caso de mujeres indígenas, la triple opresión. Aun las mujeres con recursos económicos, educativos y sociales están expuestas cotidianamente a daños, a la desatención y al maltrato conyugal, familiar, laboral y social (Sayavedra \& Flores, 1997; Fonseca \& Quintero, 2008).

Destaca el hecho de que existe una tradición sociocultural que concibe a las mujeres como cuerpo-pa- 
ra-otros, seres-objeto, seres inferiores, trasladadas a segundo plano frente a los otros y frente a las necesidades y las vidas de los otros. Las mujeres son educadas para colocarse en esa posición, a renunciar y a soportar el sufrimiento, la carencia y el dolor. Si tienen que decidir, en su mayoría sacrificarán su bienestar por los demás (Sayavedra \& Flores, 1997; Cobo, 2011).

De modo que el empoderamiento en las mujeres tiene un impacto directo en la salud. Varios estudios han demostrado que el empoderamiento femenino y la participación activa pueden tener efectos significativamente positivos en la salud y calidad de vida de su familia y comunidad (Varkey, Kureshi \& Lesnick, 2010). En este orden de ideas, si las mujeres se perciben empoderadas tendrán más control de lo que sucede en sus familias y en sus comunidades, y de esta manera propiciar su bienestar personal y social. Aquí radica la importante labor de generar programas de intervención que identifiquen áreas de oportunidad para dotar a las mujeres de herramientas de agencia personal y empoderamiento que les proporcionen mejores condiciones de vida. Este es un proceso deseable, y por supuesto necesario, pues contribuye directamente en la calidad de vida individual y familiar, a la salud y al desarrollo social (Casique, 2010).

\section{Referencias}

Bandura, A. (2000). Exercise of Human Agency Through Collective Efficacy. American Psychological Society, 9 (3), 75-78.

Bandura, A. (2005). The Primacy of Self-Regulation in Health Promotion. Applied Psychology: An International Review, 54 (2), 245-254.

Beyers. W., Goossens, L., Vansant, I., \& Moors, E. (2003). Structural Model of Autonomy in Middle and Late Adolescence: Connectedness, Separation, Detachment, and Agency. Journal of Youth and Adolescence, 32 (5), 351-365.

Brown, J. L, Pecheny, M., Gattoni, M. S., \& Tamburrino, M. C. (2013). Cuerpo, sexo y reproducción. La noción de autonomía de las mujeres puestas en cuestión: el aborto y otras situaciones sensibles. Revista Latinoamericana de Estudios sobre Cuerpos, Emociones y Sociedad, 12 (5).

Casique, I. (2010). Factores de empoderamiento y protección de las mujeres contra la violencia. Revista Mexicana de
Sociología, 72 (1), 37-71.

Charmes, J., \& Wieringa, S. (2003). Measuring Women's Empowerment: An Assessment of the Gender-Related Development Index and the Gender Empowerment Measure. Journal of Human Development, 4 (3), 419-435.

Cobo, R. (2011). Hacia una nueva política sexual. Las mujeres ante la reacción patriarcal. México: Catarata.

Doubova, V., Espinosa, P., Infante C., Aguirre, R., Rodríguez, L., Olivares, R., \& Pérez, R. (2013). Adaptación y validación de escalas de autoeficacia y empoderamiento dirigidas a mujeres mexicanas en etapa de climaterio. Salud Pública de México, 55 (3), 257-266.

Fonseca, H. C., \& Quintero. M. L. (2008). Temas emergentes en los estudios de género. México: Miguel Ángel Porrúa.

Padilla-Gámez, N., \& Cruz del Castillo, C. (2014). Construcción de una escala de vulnerabilidad antropológica y social en las mujeres. Revista de Psicología Social y Personalidad, $30(1), 13-30$.

Pick, S., Sirkin, J., Ortega, l., Osorio, P., Martínez, R., Xocolotzin, U., \& Guivaudan, M. (2007). Escala para medir agencia personal y empoderamiento (ESAGE). Revista Interamericana de Psicología. 41 (3), 295-304.

Pick, S., García, G., \& Leenen, I. (2011). Modelo para la promoción de la salud en comunidades rurales a través del desarrollo de agencia personal y empoderamiento intrínseco. Universitas Psychologica, 10 (2), 327-340.

Sánchez-Vidal, A. (2013). ¿Es posible el empoderamiento en tiempos de crisis? Repensando el desarrollo humano en el nuevo siglo. Universitas Psychologica, 12 (1), 285-300.

Sayavedra, H. G., \& Flores, H. E. (1997). Ser mujer: ¿Un riesgo para la salud? Del malestar y enfermar, al poderío y la salud. México: Red de Mujeres.

Sen, A. (1985). Well-Being, Agency and Freedom: The Dewey Lectures 1984. The Journal of Philosopy, 82 (4), 169-221.

Silva, C. L., \& Martínez, M. (2004). Empoderamiento: proceso, nivel y contexto. Psykhe, 13 (2), 29-39.

Varkey, P., Kureshi, S., \& Lesnick, T. (2010). Empowerment of Women and its Association whit the Health of the Community. Journal of Women's Health. 19 (1), 71-76.

Wilke, L. A., \& Speer, P. W. (2011). The Mediating Influence of Organizational Characteristics in the Relationship between Organizational Type and Relational Power: An Extension of Psychological Empowerment Research. Journal of Community Psychology, 39 (8), 972-986.

Zimmerman, M. A. (1995). Psychological empowerment: Issues and illustrations. American Journal of Community Psychology, 23 (5), 581-599. 
Meta-Análisis del Artículo 


\section{Dimensión Cuantitativa}

\section{Perfil de Evaluación entre pares}
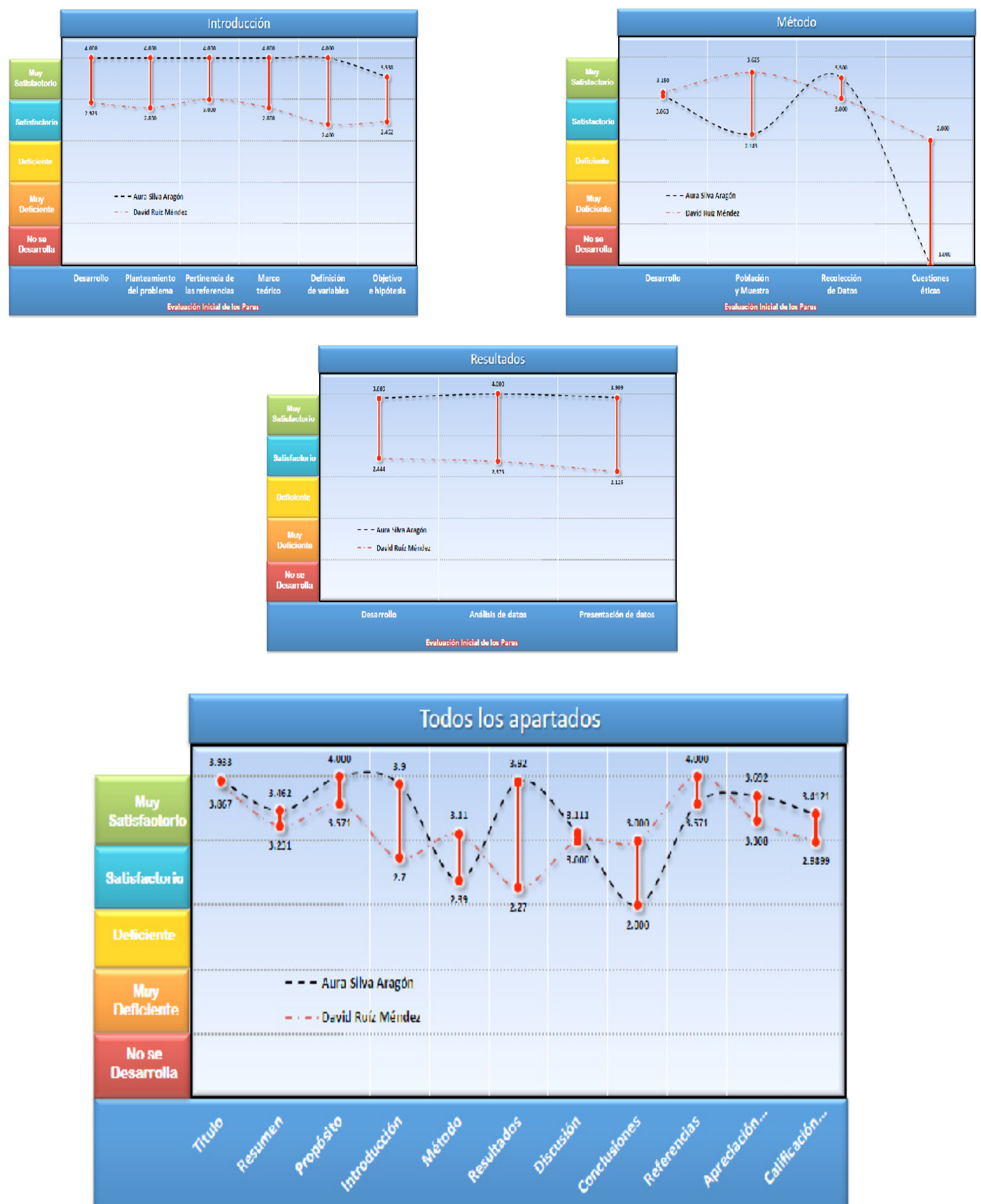

Evaluación Inicial do les Pares 


\section{Índice de Concordancia}

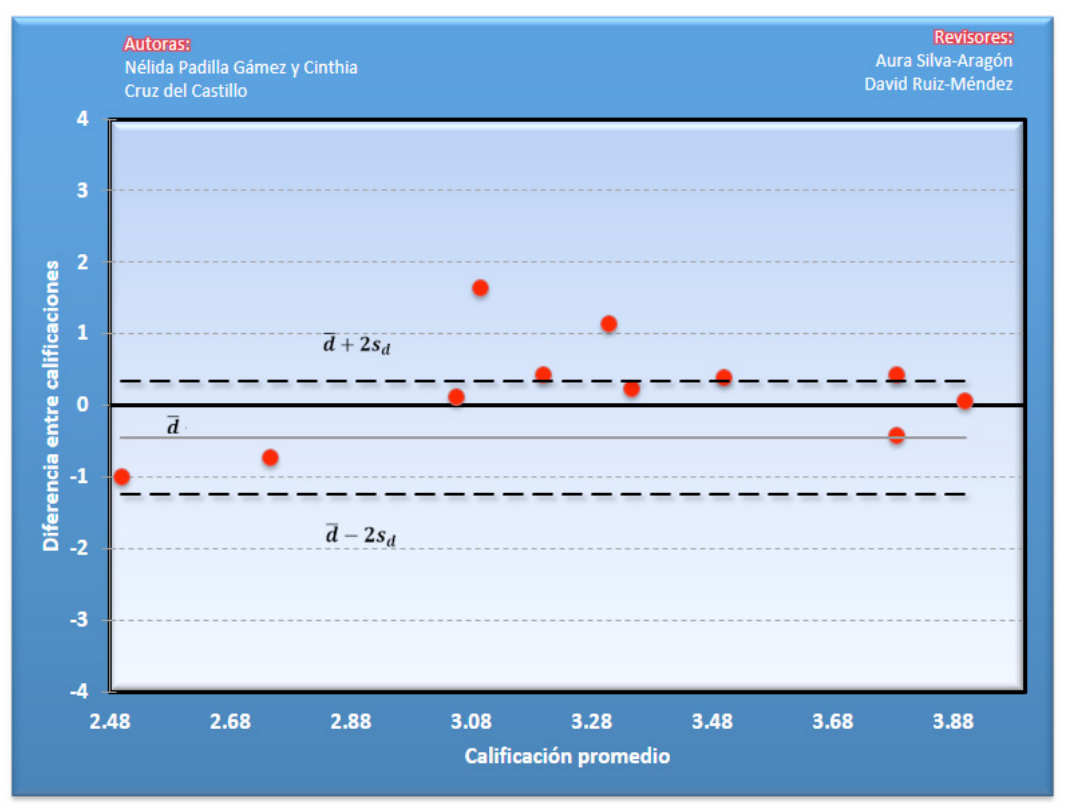

Índice de Acuerdo

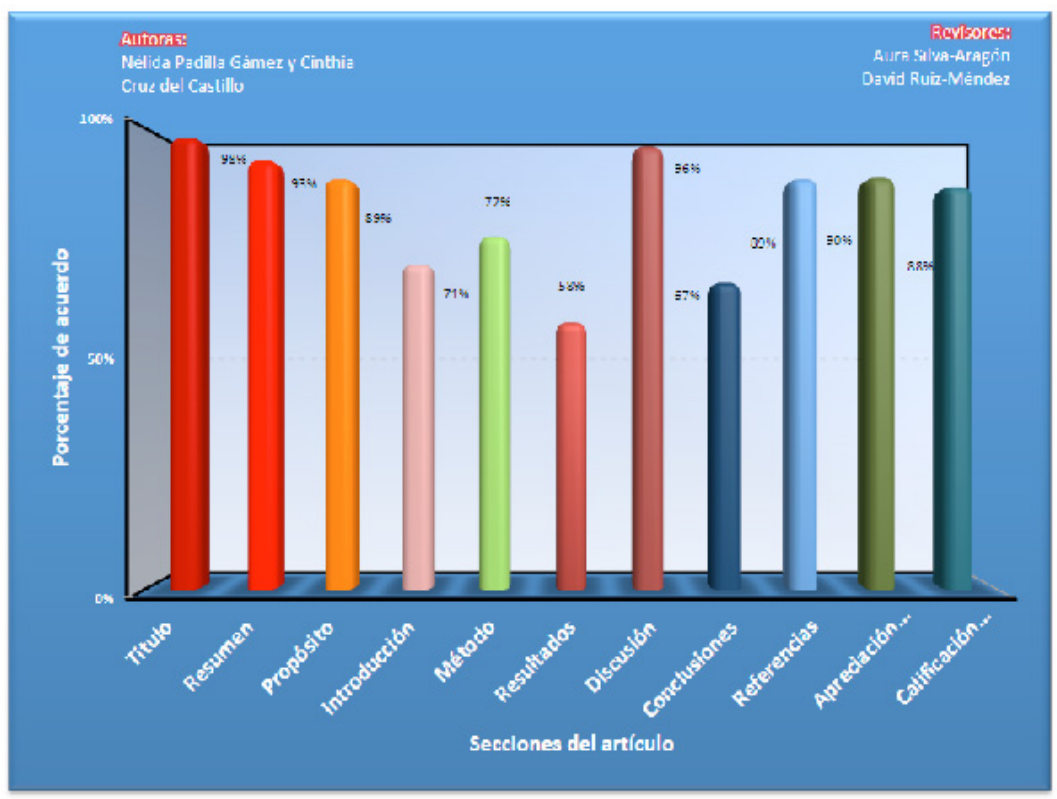


Dimensión Cualitativa

\begin{tabular}{|c|c|}
\hline Revisor 1 & Revisor 2 \\
\hline David Ruiz Méndez & Aura Silva Aragón \\
\hline \multicolumn{2}{|c|}{ Título/Autoría } \\
\hline Adecuado & $\begin{array}{l}\text { Únicamente es necesario poner el correo electrónico de } \\
\text { contacto de la segunda autora. }\end{array}$ \\
\hline \multicolumn{2}{|c|}{ Resumen } \\
\hline Adecuado & $\begin{array}{l}\text { Para mejorar la calificación en el punto } 7 \text {, tal vez sea nece- } \\
\text { sario incluir cuál fue el valor numérico de la confiabilidad } \\
\text { yla validez. En lo que respecta al punto } 10 \text {, el número total } \\
\text { de palabras del resumen es de } 188 \text {. No me parece que sea } \\
\text { un resumen muy extenso, sin embargo, no cumple con el } \\
\text { criterio. Por último, en el punto } 11 \text {, el resumen sólo pre- } \\
\text { senta } 4 \text { palabras clave, por lo que tal vez sea necesario in- } \\
\text { cluir una más para cumplir con el criterio. }\end{array}$ \\
\hline \multicolumn{2}{|c|}{ Próposito del Estudio } \\
\hline Adecuado & $\begin{array}{l}\text { En este aspecto no tengo comentarios para mejorarlo ya } \\
\text { que cumple con todos los criterios. }\end{array}$ \\
\hline \multicolumn{2}{|c|}{ Introducción } \\
\hline $\begin{array}{l}\text { Es necesario desarrollar con mayor claridad al princi- } \\
\text { pio del artículo y durante los objetivos del estudio, el } \\
\text { papel de las variables edad, presencia ausencia de hijos } \\
\text { y escolaridad. Ya que estas cobran mucha importancia } \\
\text { en las comparaciones posteriores al análisis psicomé- } \\
\text { trico del instrumento }\end{array}$ & $\begin{array}{l}\text { Considero que las hipótesis no están tan desarrolladas } \\
\text { a lo largo del marco teórico, tal vez sea importante ha- } \\
\text { cer referencia a ellas de manera más explícita. Por otra } \\
\text { parte, otra recomendación que quisiera hacer es el re- } \\
\text { dactar el contenido del artículo con lenguaje incluyente } \\
\text { debido a que el tema y la perspectiva bajo la cual se está } \\
\text { abordando lo requieren. }\end{array}$ \\
\hline
\end{tabular}




\begin{tabular}{|c|c|}
\hline Revisor 1 & Revisor 2 \\
\hline \multicolumn{2}{|c|}{ Método } \\
\hline $\begin{array}{l}\text { En la parte de análisis psicometrico es necesario desarro- } \\
\text { llar la naturaleza de cada análisis(Analisis Factorial Explo- } \\
\text { ratorio, análisis de confiabilidad de consistencia interna } \\
\text { con alfa de crombach), el orden de cada análisis, criterios } \\
\text { de análisis y pruebas previas para verificar la viabilidad del } \\
\text { AFC (valores KMO y prueba de esfericidad) En la parte de } \\
\text { comparaciones por variable (factores del instrumento) de } \\
\text { acuerdo a variables independientes, se hace primero una } \\
\text { serie de comparaciones t y luego un análisis de varianza. } \\
\text { Se recomienda quitar las comparaciones con t ya que es } \\
\text { repetitivo el análisis respecto a los ANOVA y este último } \\
\text { es mejor para controlar el error acumulado por cada par } \\
\text { de comparación (hay más de dos grupos). Donde el aná- } \\
\text { lisis con pruebas t si es adecuado, no se menciona el valor } \\
\text { de t, ni los grados de libertad, así como la relación de valor } \\
\text { de probabilidad asociado en relación al nivel de alfa esco- } \\
\text { gido (este último casi no se menciona en cada sección de } \\
\text { comparaciones) }\end{array}$ & $\begin{array}{l}\text { En cuanto al "Desarrollo" del Método, me parecería } \\
\text { importante que incluyeran un apartado de Análisis Es- } \\
\text { tadístico en el cual mencionaran cuáles fueron los es- } \\
\text { tadísticos que utilizaron para obtener la confiabilidad } \\
\text { y validez. Con respecto al tamaño de la muestra, no se } \\
\text { justifica por qué se escogió de ese tamaño, así mismo, } \\
\text { tampoco están mencionados los procedimientos para } \\
\text { la protección de los datos personales de las mujeres y } \\
\text { el consentimiento informado. Por otra parte, debido a } \\
\text { que el objetivo de la investigación fue diseñar una esca- } \\
\text { la válida y confiable, sería importante que en el método } \\
\text { mencionen cómo fue que diseñaron la escala, de dónde } \\
\text { obtuvieron los ítems o cómo los construyeron, si éstos } \\
\text { fueron sometidos a un proceso de jueceo o cómo fue } \\
\text { que decidieron cuáles y cuántos ítems conformarían la } \\
\text { escala que aplicaron. En lo que respecta a "Población y } \\
\text { Muestra" es necesario describir cuál es la población y } \\
\text { después justificar el tamaño de la muestra en función } \\
\text { de los objetivos de la investigación. En la "Recolec- } \\
\text { ción de Datos" sería importante que describan de for- } \\
\text { ma más clara cuál fue el procedimiento que utilizaron } \\
\text { para acercarse a las mujeres que participaron en la in- } \\
\text { vestigación. Por último, considero que la investigación } \\
\text { no desarrolla ninguna información con respecto a las } \\
\text { "Consideraciones Éticas", por lo tanto, es importante } \\
\text { incluir cuáles fueron las medidas que siguieron para la } \\
\text { protección de datos personales, asegurar el anonimato } \\
\text { y el procedimiento de consentimiento informado. }\end{array}$ \\
\hline \multicolumn{2}{|c|}{ Resultados } \\
\hline $\begin{array}{l}\text { No se incluyen los valores de probabilidad asociada de } \\
\text { acuerdo a cada análisis. En algunos casos, hay análisis re- } \\
\text { dundantes (pares de pruebas t vs ANOVAS). Las tablas } \\
\text { que indican las medias no contienen los valores de F o t n } \\
\text { indican su significación en términos del nivel de alfa esco- } \\
\text { gido. Las correlaciones de pearson no carecen de valor de } \\
\text { probabilidad asociado, a pesar de que se realizó el análisis } \\
\text { de inferencia estadística }\end{array}$ & $\begin{array}{l}\text { La sección de Resultados es muy adecuada, únicamente } \\
\text { recomendaría que las explicaciones y argumentos se omi- } \\
\text { tieran ya que este apartado debe ser meramente descrip- } \\
\text { tivo. Toda la argumentación de esta sección puede ser in- } \\
\text { cluida en la Discusión. }\end{array}$ \\
\hline
\end{tabular}




\begin{tabular}{|c|c|}
\hline Revisor 1 & Revisor 2 \\
\hline \multicolumn{2}{|c|}{ Discusión } \\
\hline Adecuado & $\begin{array}{l}\text { En el apartado de Discusión sería importante incluir re- } \\
\text { sultados de otros estudios para comparar y contrastar con } \\
\text { los resultados de esta investigación, así mismo, es necesa- } \\
\text { rio discutir las limitaciones que tuvo la investigación. }\end{array}$ \\
\hline \multicolumn{2}{|c|}{ Conclusiones } \\
\hline Adecuado & $\begin{array}{l}\text { El artículo no posee un apartado de "Conclusiones". Los } \\
\text { puntos que fueron calificados con } 4 \text { es porque se incluyen } \\
\text { en la Discusión, sin embargo, sería importante incluir la } \\
\text { sección de Conclusiones para poder desarrollar todos los } \\
\text { puntos que no están presentes. }\end{array}$ \\
\hline \multicolumn{2}{|c|}{ Referencias } \\
\hline Adecuado & $\begin{array}{l}\text { Las referencias son adecuadas, sin embargo, la mayoría de } \\
\text { ellas no son de los últimos } 5 \text { años. Así mismo, tal vez po- } \\
\text { dría incluir algunas más para cumplir con el criterio del } \\
\text { punto } 3 \text {. }\end{array}$ \\
\hline
\end{tabular}


